\title{
Effect of intrathecal injection of miRNA-138 on neuropathic pain in rats undergoing partial sciatic nerve ligation and its underlying mechanism
}

\author{
Yantao Jin ${ }^{1}$, Lili $\mathrm{Xu}^{2}$, Yanrong $\mathrm{Xu}^{3}$ \\ ${ }^{1}$ Department of Anesthesiology, Tangshan Workers' Hospital, Tangshan, China; ${ }^{2}$ Department of Anesthesiology, Tangshan Hongci Hospital, \\ Tangshan, China; ${ }^{3}$ Department of Anesthesiology, Tangshan Centrail Hospital, Tangshan, China \\ Contributions: (I) Conception and design: Y Jin; (II) Administrative support: Y Jin; (III) Provision of study materials or patients: L Xu; (IV) Collection \\ and assembly of data: L Xu; (V) Data analysis and interpretation: Y Xu; (VI) Manuscript writing: All authors; (VII) Final approval of manuscript: All \\ authors. \\ Correspondence to: Yanrong Xu. Department of Anesthesiology, Tangshan Centrail Hospital, Youyi Road and Changning Road intersection, Lubei \\ District, Tangshan, China. Email: xyrjyt@163.com.
}

\begin{abstract}
Background microRNA-138 (miRNA-138) might have a promising therapeutic effect in the Neuropathic pain (NP). We aim to investigate the effects of miRNA-138 on NP and explore its underlying mechanism.

Methods: we performed a partial sciatic nerve ligation (pSNL) surgery in rats to induce pain and inflammation. Rats were administrated by intrathecal injection of lentiviral (LV)-mediated miRNA-138. Mechanical withdrawal threshold (MWT) and paw withdrawal thermal latency (PWTL) were measured to evaluate the pain degree. The expression levels of miRNA-138, toll-like receptor 4 (TLR4), tumor necrosis factor-alpha (TNF- $\alpha$ ), interleukin- $\beta$ (IL- $\beta$ ), and IL-6 in the spinal cord were detected by quantitative realtime polymerase chain reaction (qRT-PCR). Western blotting was performed to measure the expressions of macrophage inflammatory protein-1 alpha (MIP-1 $\alpha$ ) and C-C chemokine receptor type 1 (CCR1). Next, the mechanism of miRNA-138 on NP was investigated by intrathecal injection of CCR1 inhibitor or MIP- $1 \alpha$ neutralizing antibody. Inflammatory factors, MWT, and PWTL were also measured on day 7.

Results: Intrathecal injection of miRNA138 significantly reduced MWT and PWTL. qRT-PCR showed that miRNA138 mimic group significantly reduced the level of TLR4, TNF- $\alpha$, Il- $\beta$, and IL-6 on day 7. Western blotting showed that the protein expressions of MIP-1 $\alpha$ and CCR1 in pSNL + miRNA138 mimic group were significantly decreased on day 7 . In addition, the miRNA138 inhibitor inversely increased MWT, PWTL and inflammatory cytokines. Further, the effect of miRNA138 inhibitor all were significantly reversed by CCR1 inhibitor or MIP-1 $\alpha$ neutralizing antibody.
\end{abstract}

Conclusions: Intrathecal injection of miRNA-138 can remarkably alleviate NP in rats with a pSNL, which may be achieved by suppressing the TLR4 and MIP-1 $\alpha / C C R 1$ signaling pathways.

Keywords: MicroRNA-138; neuropathic pain (NP); toll-like receptor 4 (TLR4); neuroinflammation; MIP-1 $\alpha /$ CCR1

Submitted Jan 26, 2021. Accepted for publication May 21, 2021.

doi: 10.21037/apm-21-669

View this article at: http://dx.doi.org/10.21037/apm-21-669

\section{Introduction}

Neuropathic pain (NP) is a common chronic pain caused by nerve dysfunction or lesions $(1,2)$, which routine analgesics often fail to meaningfully resolve. NP is typically featured by hypersensitivity of nociceptive neurons in the central and peripheral nervous systems $(3,4)$. Inflammation and glial cell activation have been reported to be important mechanisms involved in NP, and therefore blocking the inflammatory 
cascade is considered an important strategy to relieve NP (5).

MicroRNAs (miRNAs) are a class of endogenously expressed small, noncoding, single-stranded RNA that regulate gene expression by inhibiting the translation or promoting the degradation of target messenger RNAs (mRNAs) (5). Many recent studies have demonstrated the dysregulation of miRNA expression in human diseases including tumors, intervertebral disc degeneration, diabetes, and coronary artery disease $(6,7)$. miRNA-138 is specifically highly expressed in the nervous system, and alterations in its expression levels are closely associated with the development of several neurological disorders. Studies have shown that miRNA-138 plays an important role in a variety of cellular biological processes and that its expression is associated with the initiation and maintenance of various diseases, suggesting upregulation of miRNA-138 may be beneficial (8). In addition, miRNA-138 has been shown to alleviate traumatic brain injury (TBI)-induced brain damage and suppress breast cancer and non-small cell lung cancer (9-11). Toll-like receptor 4 (TLR4), a key member in the TLR family, is a highly conserved innate immune receptor that is expressed in the central nervous system, primarily in microglia that cause inflammatory activation (12). TLR4 can contribute to the development and progression of a variety of diseases, including NP $(13,14)$. Multiple immune and glial cells have been reported to release macrophage inflammatory protein alpha (MIP-1 $\alpha$ ), a regulator of inflammatory pain and nociceptive hypersensitivity, which regulates inflammation and the nervous system by activating C-C chemokine receptor 1 (CCR1), the receptor for MIP$1 \alpha$ (15). However, the roles of miRNA-138 and TLR4 as well as MIP-1 $\alpha /$ CCR 1 in NP have not been well elucidated. Here we explored the roles of miRNA-138 and TLR4 in $\mathrm{NP}$ and neuroinflammation, with an attempt to further inform the effective treatment of NP.

We present the following article in accordance with the ARRIVE reporting checklist (available at http://dx.doi. org/10.21037/apm-21-669).

\section{Methods}

\section{Materials}

\section{Experimental animals}

Sprague-Dawley (SD) rats (male, weighing 210-300 g) were used. All experimental protocols were approved by the ethics committee of Laboratory Animal Center, Hebei Medical University (approval No. 20200912) and were performed in accordance with the Guidelines for the Care and Use of Laboratory Animals.

\section{Reagents and instruments}

Lidocaine was purchased from Sigma (Sigma-Aldrich, St. Louis, MO, USA); isoflurane was purchased from Hebei Yipin Pharmaceutical (Shijiazhuang, Hebei, China); TLR4, TNF- $\alpha$, IL- $\beta$, IL- 6 , GADPH, MIP- $1 \alpha$, and goat anti-rabbit immunoglobin $\mathrm{G}$ ( $\mathrm{IgG}$ )-horseradish peroxidase (HRP) antibodies were purchased from Cell Signaling Technology (CST; Danvers, MA, USA); MIP- $1 \alpha$ Ab was purchased from R\&D Systems, Inc. (Minneapolis, MN, USA) and CCR1-HY from MCE (Shanghai, China); tissue lysate was purchased from Beyotime Tiotechnology (Shanghai, China); electrophoresis instrument, electrophoresis tank, and electrotransfer membrane system were purchased from Bio-Rad Laboratories (Hercules, CA, USA); a TRIZOL kit was purchased from Invitrogen (Carlsbad, CA, USA); PE-10 polyethylene tubing was purchased from American Research Products (Grandville, MI, USA); electronic von Frey aesthesiometer was purchased from Stoelting (Wood Dale, IL, USA); a thermal-tingling apparatus (PL-200) was purchased from Taimeng (Chengdu, China). All the lentiviruses for injection and their controls were purchased from Genepharma (Suzhou, China).

\section{Study methods}

\section{Animal grouping}

Twenty-four healthy adult male SD rats were randomly divided into three groups: a normal group (without any treatment), a sham group (exposure of the sciatic nerve but without ligation), and a partial sciatic nerve ligation (pSNL) group. In addition, 32 healthy adult male SD rats were randomly divided into four groups: a sham + miRNA-138negative control (NC) group (sham operation + intrathecal injection of LV-miRNA-138-NC), a pSNL + miRNA-138NC group (pSNL + intrathecal injection of LV-miRNA138-NC), a sham + miRNA-138 mimic group (sham operation + intrathecal injection of LV-miRNA-138), and a pSNL + miRNA-138 mimic group (pSNL + intrathecal injection of LV-miRNA-138). Finally, 48 healthy adult male SD rats were randomly divided into 6 groups: a sham group (sham operation), a pSNL + miRNA-138$\mathrm{NC}$ group [pSNL + intrathecal injection of lentivirus (LV)-miRNA-138-NC], a pSNL + miRNA-138 mimic group (pSNL + intrathecal injection of LV-miRNA-138), a pSNL + miRNA-138 inhibitor group (pSNL + intrathecal 
injection of LV-miRNA-138 inhibitor), a pSNL + miRNA-138 inhibitor + MIP- $1 \alpha$ Ab group (pSNL + intrathecal injection of LV-miRNA-138 inhibitor + MIP- $1 \alpha$ neutralizing antibody), and a pSNL+miRNA-138 inhibitor + CCR1-HY group (pSNL + intrathecal injection of LV-miRNA-138 inhibitor + CCR1 inhibitor). All animals were housed under standard laboratory conditions and kept under constant environmental conditions $\left(21-26^{\circ} \mathrm{C}, 40-60 \%\right.$ humidity, and a 12-hour day light-dark cycle). Food and water were offered ad libitum. To not disturb the biological rhythm and behavior of the animals, the experiments were performed between 9 am and $6 \mathrm{pm}$.

\section{Establishment of animal models of pSNL}

Rats were anesthetized with isoflurane (Hebei Yipin Pharmaceutical, Shijiazhuang, China) by inhalation. Under aseptic conditions, the right sciatic nerve was exposed by an incision from the right sciatic notch to the distal thigh. With the femoral head as a landmark, the location of the sciatic nerve ligation was determined. Approximately half of the sciatic nerve was tightly ligated with a 7-0 silk suture (Merck Millipore, Billerica, MA, USA). The incision was closed with 5-0 cotton suture (Merck Millipore) and disinfected with ethanol (Hebei Yipin Pharmaceutical,). In the sham-operated groups, the right-side sciatic nerve was exposed but not ligated.

\section{Intrathecal injection of LV-miRNA-138}

The rats were anesthetized with an intraperitoneal injection of chloral hydrate (Hebei Yipin Pharmaceutical,). At an angle of approximately $20^{\circ}$ above the vertebral column, the PE-10 catheter was inserted into the intervertebral space between the L5 and L6 spinous processes, entering the trough-like space between the transverse process and spinous process. A sudden slight flick of the tail or paw indicated successful entry into the dorsal subarachnoid space. Implantation of intrathecal catheter was then performed, followed by fixing of the catheter and sealing of the incision. The pSNL surgery was performed 2 days later. One day before surgery, the recombinant LV-miRNA-138 mimic (Genepharma) or miRNA-138-NC (Genepharma) was slowly injected over 1 minute using a microinjection syringe (Merck Millipore,) attached to the intrathecal catheter.

\section{Mechanical withdrawal threshold (MWT) and paw withdrawal thermal latency (PWTL) measurements} MWT and PWTL were measured on days $0,3,7,14$, and 21.
For MWT measurement, the rats were housed in a cage with sufficiently large space to allow free movement. All the cages had mesh flooring. The room temperature was kept constant during the experiments to avoid any disturbance from external factors. The rats were placed in the cage within 1 hour prior to the experiment to make them acclimatize to environmental stresses. A calibrated von Frey filament (Stoelting Co., Wood Dale, IL, USA) was applied starting in ascending order to the plantar surface of the hind paw for approximately 5 seconds. The force that induced paw withdrawal was recorded. The measurements repeated were4in 5 sessions, and each session lasted no more than 5 seconds. After the extremes were removed, the average value was considered to be the PWT.

For PWTL measurement, the plantar surface of the hind-paw was exposed to the vertical thermal radiation on the thermal-tingling apparatus (PL-200, Taimeng, Chengdu, China). Monitoring was required during the radiation to avoid thermal damage to the plantar surface. The withdrawal latency of the left hind limb was recorded during the thermal radiation. The measurements were repeated in eive sessions, with an interval of 5 seconds between two sessions. During the consecutive tests, the hind paws were tested alternately at intervals greater than 3 minutes. The PWTL value of the rats was averaged after removing the extreme values.

Detection of the expressions of miRNA-138, TLR4, tumor necrosis factor- $\alpha$, interleukin- $\beta$, and interleukin-6 by quantitative real-time polymerase chain

The rats were anesthetized with isoflurane and then sacrificed by spinal cord transection on day 7 . The skin and subcutaneous tissues were incised along the spinous process, and the dorsal horns of the lateral lumbar $\left(\mathrm{L}_{4-6}\right)$ were rapidly excised. Total RNA was extracted using Trizol (Thermo Fisher Scientific, USA), following the manufacturer's protocol. Complement DNA (cDNA) was synthesized using a reverse transcription system. The polymerase chain reaction (PCR) primers were designed and synthesized by Sangon Biotech (Shanghai, China; Table 1). U6 was used for the normalization of miRNA, and GAPDH and U6 were used as internal controls for mRNA expression. Amplification was performed by quantitative real-time polymerase chain (qRT-PCR), and each group was tested 3 times in duplicate. The expression levels of miRNA-138, TLR4, tumor necrosis factor- $\alpha$, (TNF- $\alpha$ ), interleukin- $\beta$ (IL- $\beta$ ), and IL-6 were analyzed by the $2^{-\Delta \Delta C t}$ method. 
Table 1 Primer sequences for qRT-PCR

\begin{tabular}{ll}
\hline Gene name & Primer sequence (5'-3') \\
\hline miRNA-138 & Forward: 5'-GGTGTCCGTGGAGTCGGCAA-3' \\
& Reverse: 5'-AACTTCACAACACCAGCTTA-3' \\
GAPDH & Forward: 5'-AAGGGAAGGTTGCTGGATAGG-3' \\
& Reverse: 5'-CACATCCACCTCCTCCACATC-3' \\
U6 & Forward: 5'-CTCGCTTCGGCAGCACA-3' \\
& Reverse: 5'-AACGCTTCACGAATTTGCGT-3' \\
TLR4 & Forward: 5'-GTTCCTGACACTACTACAAGATTC-3' \\
& Reverse: 5'-GCTGGAGCAGATGAGAGT-3' \\
IL- $\beta$ & Forward: 5'-CTGGCGCTCAGCCATACAG-3' \\
& Reverse: 5'-CGCACTTATACTGGTCAAATCCC-3' \\
IL-6 & Forward: 5'-GACTGATGTTGTTACAGCCACTGC-3' \\
& Reverse: 5'-AGCCACTCCTTCTGTGACTCTAACT-3' \\
& Forward: 5'-CATGATCCGAGATGTGGAACTGGC-3' \\
TNF- $\alpha$ & Reverse: 5'-CTGGCTCAGCCACTCCAGC-3' \\
\hline & \\
\hline
\end{tabular}

Detection of MIP-1 $\alpha$ and CCR1 protein expressions by western blotting

On day 7, $\mathrm{L}_{4-6}$ spinal cord tissues were harvested as described in qRT-PCR experiments, lysed with RIPA buffer containing protease inhibitor cocktail (Thermo Fisher Scientific) and phosphatase inhibitor (Thermo Fisher Scientific), and then centrifuged at low temperature for 5 minutes $\left(4^{\circ} \mathrm{C}, 12,000 \mathrm{rpm}\right)$. The protein concentrations were determined by bicinchoninic acid (BCA) method. A total of $40 \mu \mathrm{g}$ of total protein was denatured by boiling with sodium dodecyl sulfate (SDS) sample buffer (Merck Millipore) and then loaded and separated by $10 \%$ SDSPAGE (Merck Millipore). After electrophoresis, proteins were transferred to polyvinylidene fluoride (PVDF) membranes (no. LC2002, Thermo Fisher Scientific). The PVDF membranes were placed in $5 \%$ skimmed milk powder (BD Biosciences, San Jose, CA, USA), sealed at room temperature for 2 hours, and then incubated with MIP- $1 \alpha$ and CCR1 primary antibodies (CST; 1:1,000) overnight at $4{ }^{\circ} \mathrm{C}$. The mixture was incubated with goat anti-rabbit antibody (CST; 1:10,000) at room temperature for 2 hours. In a dark room, the PVDF membranes (Merck Millipore) were placed in a dark box, and luminescent liquid (Merck Millipore) was added dropwise. Image Lab v.3.0 software (Bio-Rad Laboratories) was used to acquire and analyze imaging signals. The expression levels of the target proteins were calculated against GAPDH levels.

\section{Statistical analysis}

All data were analyzed using the SPSS 21.0 software (IBM Corp., Armonk, NY, USA). The normally distributed measurement data are presented using mean \pm standard deviation $(\bar{x} \pm \mathrm{SD})$. Multiple groups were compared using one-way analysis of variance (ANOVA), and the comparisons between two groups were based on the $t$ test. A $P$ value of $<0.05$ was considered statistically significant.

\section{Results}

miRNA-138 and TLR4 expression levels in the spinal cord after $p S N L$

Spinal cord tissues were excised from each group on days 3 , 7, 14, and 21; total RNA was extracted; and the expression levels of miRNA-138 and TLR4 in the L4-6 lateral lumbar cords were measured by qRT-PCR. qRT-PCR revealed that miRNA-138 was significantly downregulated in the pSNL group compared with the normal group and the sham group on day $3(\mathrm{t}=8.79, \mathrm{P}<0.05)$ and continued to decrease over time until day $21(\mathrm{t}=10.31, \mathrm{P}<0.05$; Figure $1 A)$. The mRNA expression of TLR4 was significantly upregulated in the pSNL group on day $3(\mathrm{t}=7.49, \mathrm{P}<0.05$; Figure $1 B)$ and continued to rise until day $21(\mathrm{t}=8.14, \mathrm{P}<0.05$; Figure $1 B)$.

\section{MWT and PWTL in rats after pSNL}

The miRNA-138 expression was significantly downregulated in the spinal cord tissue on the surgical side in pSNL rats, indicating a potential correlation between miRNA-138 expression and NP in pSNL rats. To further investigate the biological role of miRNA-138 in NP, miRNA-138 expression in rat spinal cord tissue was upregulated by intrathecal injection of $\mathrm{LV}$, which mediated miRNA-138 infection (miRNA-138 mimic). MWT and PWTL were measured continuously on days $0,3,7,14$, and 21 to evaluate the effect of upregulated miRNA-138 on NP. On day 7, the MWT and PWTL values in the PSNL + miRNA-138 mimic group were significantly higher than those in the pSNL + miRNA-138-NC group $(13.50 \pm 0.34 \mathrm{vs}$. $10.50 \pm 0.52 \mathrm{~g}, \mathrm{t}=12.61, \mathrm{P}<0.05 ; 15.50 \pm 0.73$ vs. $9.50 \pm 0.33 \mathrm{~s}$, $\mathrm{t}=4.99, \mathrm{P}<0.05$; Figure $2 A, B)$. On day 21 , the MWT value decreased to $5.87 \pm 0.67 \mathrm{~g}$ and the PWTL value decreased to $8.87 \pm 0.67 \mathrm{~s}$ in the pSNL + miRNA-138 mimic group, 
A

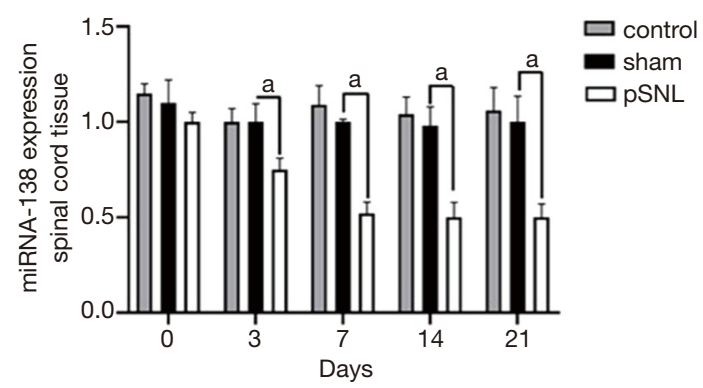

B

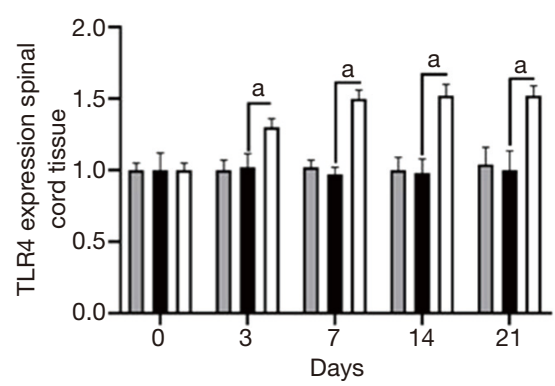

Figure 1 mRNA expression levels of miRNA-138 and TLR4 in $\mathrm{L}_{4-6}$ spinal cord tissues of rats in each group on days 3, 7, 14, and 21 $(\mathrm{n}=5)$. (A) mRNA expression of miRNA-138 in each group (by qRT-PCR). (B) mRNA expression of TLR4 in each group (by qRTPCR). ${ }^{\text {a }}, \mathrm{P}<0.05$.

A

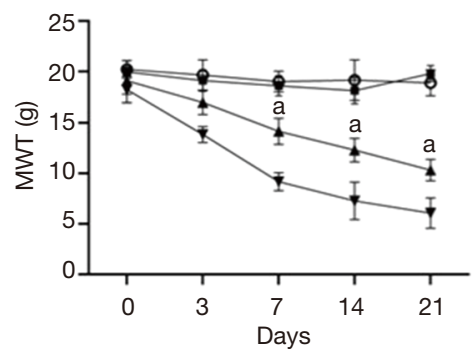

B

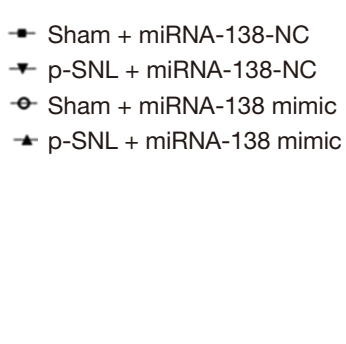

Figure 2 miRNA-138 overexpression alleviated the development of neuropathic pain in pSNL rats ( $\mathrm{n}=5$ ). (A) Change of MWT on days 0,3 , 7, 14, and 21 in each group. (B) Change of PWTL in each group. ", $\mathrm{P}<0.05$. Sham + miRNA-138-NC group: sham-operated rats transfected with miRNA-138-NC; pSNL+miRNA-138-NC group: pSNL rats transfected with miRNA-138-NC; sham + miRNA-138 mimic group: sham-operated rats transfected with miRNA-138 mimic; pSNL + miRNA-138 mimic group; pSNL rats transfected with miRNA-138.

which remained significantly higher than those in the $\mathrm{pSNL}+$ miRNA-138-NC group $(\mathrm{t}=17.35, \mathrm{P}<0.05 ; \mathrm{t}=6.47$, $\mathrm{P}<0.05)$. It was therefore assumed that intrathecal injection of miRNA-138 significantly suppressed thermal and mechanical nociceptive responses in pSNL rats.

\section{miRNA-138 overexpression decreased the expressions of inflammation-related factors including TLR4 and TNF- $\alpha$ in pSNL rats}

We found that miRNA-138 expression was decreased and TLR4 expression was increased in pSNL rats from day 3 to day 21. To further explore the effects of miRNA-138 on inflammation-related factors including TLR4, TNF- $\alpha$, IL$\beta$, and IL- 6 in the spinal cord of pSNL rats, we examined the changes of TLR4, TNF- $\alpha$, IL- $\beta$, and IL-6 expressions in the spinal cord of pSNL rats intrathecally injected with miRNA-138 on day 7. qRT-PCR confirmed the successful expression of miRNA-138, evidenced by that the pSNL + miRNA138 mimic group had a significantly higher expression of miRNA-138 than pSNL + miRNA138$\mathrm{NC}$ group $(\mathrm{t}=9.56, \mathrm{P}=0.023)$. Also, compared with the pSNL + miRNA138-NC group, the pSNL + miRNA138 mimic group had significantly lower expressions of TLR4, TNF- $\alpha$, Il- $\beta$, and IL- 6 on the postoperative day $6(t=5.32$, $\mathrm{P}<0.05 ; \mathrm{t}=7.69, \mathrm{P}<0.05 ; \mathrm{t}=6.35, \mathrm{P}<0.05 ; \mathrm{t}=8.15, \mathrm{P}<0.05$; Table 2). Thus, intrathecal injection of miRNA-138 might suppress nerve pain in rat models of pSNL and ultimately downregulate inflammation-related factors by decreasing TLR4, suggesting that TLR4 might be a potential target gene for miRNA-138.

\section{miRNA-138 inbibited the MIP-1a/CCR1 signaling patbway in $\mathrm{pSNL}$ rats}

It has been previously reported that the MIP-1a/ 
Table 2 Relative expression levels of inflammatory factors $(\bar{x} \pm \mathrm{SD}, \mathrm{n}=8)$

\begin{tabular}{|c|c|c|c|c|c|c|}
\hline Group & Sham + miRNA-138-NC & $\mathrm{pSNL}+\mathrm{miRNA}-138-\mathrm{NC}$ & Sham + miRNA-138 mimic & $\mathrm{pSNL}+$ miRNA-138 mimic & $\mathrm{t}$ & $P$ \\
\hline TLR4 & $1.10 \pm 0.17$ & $3.31 \pm 0.39$ & $1.89 \pm 0.18$ & $2.87 \pm 0.27^{\mathrm{a}}$ & 5.32 & 0.048 \\
\hline TNF- $\alpha$ & $1.01 \pm 0.21$ & $4.32 \pm 0.42$ & $1.78 \pm 0.11$ & $2.64 \pm 0.20^{\mathrm{a}}$ & 7.69 & 0.030 \\
\hline IL- $\beta$ & $1.04 \pm 0.16$ & $4.87 \pm 0.30$ & $1.36 \pm 0.16$ & $2.53 \pm 0.22^{\mathrm{a}}$ & 6.35 & 0.037 \\
\hline
\end{tabular}

qRT-PCR detected the expressions of miRNA-138, TLR4, TNF- $\alpha$, IL- $\beta$, and IL- 6 in $\mathrm{L}_{4-6}$ lateral lumbar cords of rats in each group on day 7 . ${ }^{a}, \mathrm{P}<0.05$, compared with the pSNL+ miRNA-138-NC group. Sham + miRNA-138-NC group: sham-operated rats transfected with miRNA138-NC; pSNL + miRNA-138-NC group: pSNL rats transfected with miRNA-138-NC; sham + miRNA-138 mimic group: sham-operated rats transfected with miRNA-138 mimic; pSNL+ miRNA-138 mimic group; $p S N L$ rats transfected with miRNA-138.

A

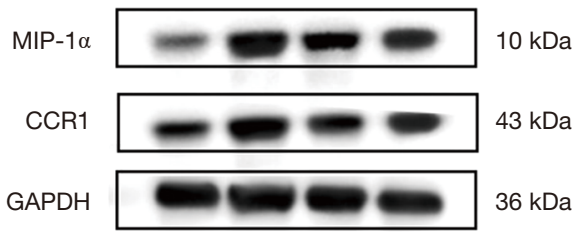

B

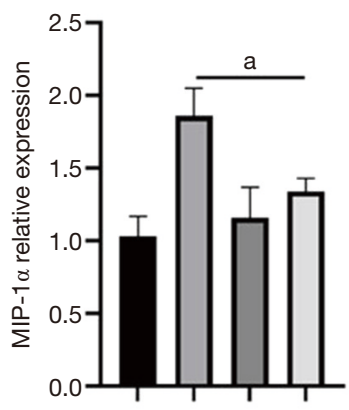

C

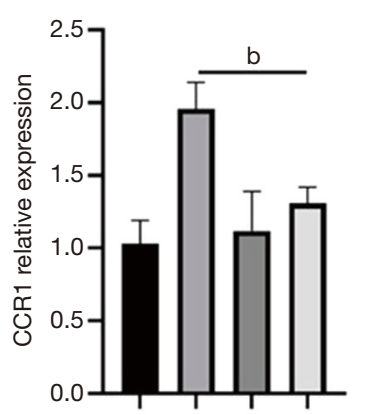

Sham + miRNA-138-NC

$\square \mathrm{pSNL}+$ miRNA-138-NC

$\square$ Sham + miRNA-138 mimic

$\square$ pSNL + miRNA-138 mimic

Figure 3 miRNA-138 affected the progression of neuropathic pain via MIP-1 $\alpha$ and CCR1 in pSNL rats (n=5). (A) WB blot of MIP-1 $\alpha$ and CCR1. (B) MIP-1 $\alpha$ protein expression in the $\mathrm{L}_{4-6}$ spinal cord of rats in each group on day 7. (C) CCR1 protein expression in the $\mathrm{L}_{4-6}$ spinal cord of rats in each group on day 7 , $^{\mathrm{a}}, \mathrm{P}<0.05 ;{ }^{\mathrm{b}}, \mathrm{P}<0.01$. Sham + miRNA-138-NC group: sham-operated rats transfected with miRNA-138NC; pSNL + miRNA-138-NC group: pSNL rats transfected with miRNA-138-NC; sham + miRNA-138 mimic group: sham-operated rats transfected with miRNA-138 mimic; pSNL + miRNA-138 mimic group; pSNL rats transfected with miRNA-138.

CCR1 signaling pathway is involved in neuralgia and neuroinflammation (10). On day 7, we detected the protein expression levels of MIP-1 $\alpha$ and CCR1 in the spinal cord of rats in four groups (Figure 3). Western blotting showed that the protein expressions of MIP- $1 \alpha$ and CCR1 in the pSNL + miRNA138 mimic group were significantly decreased on day $7(\mathrm{t}=7.75, \mathrm{P}<0.05 ; \mathrm{t}=6.97, \mathrm{P}<0.05)$ compared with the pSNL + miRNA-138-NC group.

\section{Interference with MIP-1 1 /CCR1 signaling patbway ameliorated miRNA-138-induced neuroinflammation and NP}

To verify the roles of MIP- $1 \alpha$ and CCR1 in this process, we injected a miRNA-138 inhibitor, a neutralizing antibody, to MIP-1 $\alpha$ (MIP-1 $\alpha \mathrm{Ab}$ ), and an inhibitor of CCR1 (CCR1$\mathrm{HY}$ ), respectively, into the myelin sheath of rats in six groups (Figure $4 A, B, C, D$ ). On day $7, \mathrm{MWT}$ and PTWL 
A

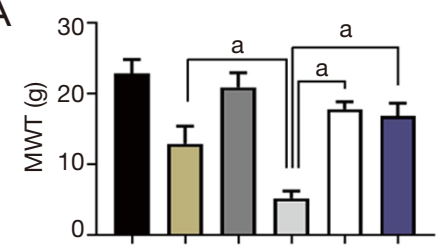

B

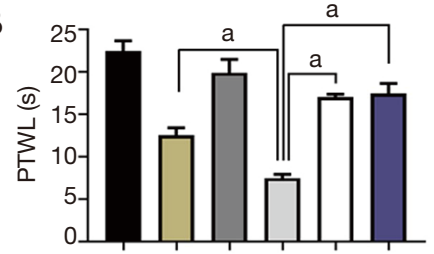

C

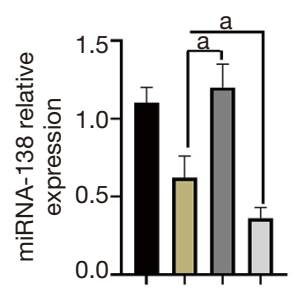

D

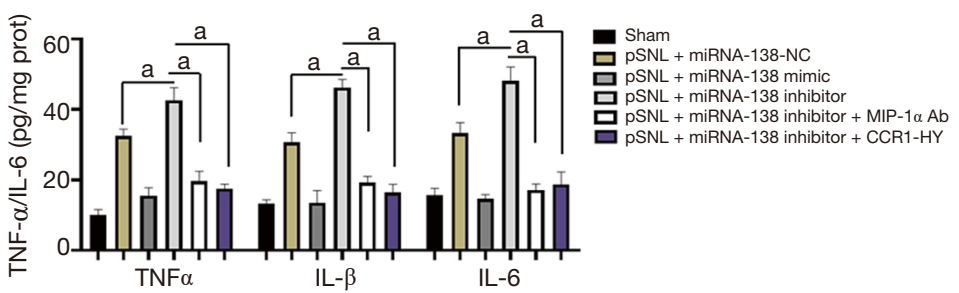

Figure 4 Interference with MIP-1 $\alpha$ /CCR1 signaling pathway ameliorated miRNA-138-induced neuroinflammation and NP. (A) MWT on day 7. (B) PTWL on day 7. (C) Expressions of miRNA-138 in $\mathrm{L}_{4-6}$ lateral lumbar cords of rats in each group on day 7 (by qRTPCR). (D) Expressions of TLR4, TNF- $\alpha$, IL- $\beta$, and IL-6 in L $_{4-6}$ lateral lumbar cords of rats in each group on day 7 (by qRT-PCR). a, $\mathrm{P}<0.05 . \mathrm{pSNL}+$ miRNA-138-NC group: pSNL rats transfected with miRNA-138-NC; pSNL + miRNA-138 inhibitor group: pSNL rats transfected with miRNA-138 inhibitor; pSNL+miRNA-138 inhibitor + MIP-1 $\alpha$ Ab group: pSNL rats transfected with pSNL + miRNA-138 inhibitor combined with MIP-1 $\alpha$ neutralizing antibody; pSNL + miRNA-138 inhibitor + CCR1-HY group: pSNL rats transfected with miRNA-138 inhibitor and CCR1 inhibitor.

were measured and qRT-PCR was performed in these 6 groups (Figure $4 A, B)$. Compared with the pSNL + miRNA138-NC group, both MWT $(\mathrm{t}=7.84, \mathrm{P}<0.05)$ and PTWL $(\mathrm{t}=4.99, \mathrm{P}<0.05)$ in the $\mathrm{pSNL}+$ miRNA-138 inhibitor group were significantly increased. Compared with the pSNL + miRNA-138 inhibitor group, both the pSNL + miRNA-138 inhibitor + MIP-1 $\alpha$ Ab group $(t=9.51$, $\mathrm{P}<0.05 ; \mathrm{t}=10.68, \mathrm{P}<0.05)$ and the $\mathrm{pSNL}+$ miRNA-138 inhibitor + CCR1-HY group $(\mathrm{t}=16.34, \mathrm{P}<0.05 ; \mathrm{t}=16.98$, $\mathrm{P}<0.05)$ had significantly increased MWT and PTWL. The higher expression of miRNA-138 in the pSNL + miRNA-138-mimic group than pSNL + miRNA138$\mathrm{NC}$ group indicated the efficacy of transfection in the rats $(\mathrm{t}=11.61, \mathrm{P}<0.05)$. Unsurprisingly, pSNL + miRNA-138 inhibitor group showed lower expression of miRNA-138 than $\mathrm{pSNL}+$ miRNA138-NC group $(\mathrm{t}=10.73, \mathrm{P}<0.05$, Figure $4 C$ ). qRT-PCR revealed that injection of LVmiRNA-138 inhibitor upregulated the expressions of TNF- $\alpha$, IL- $\beta$, and IL-6 in rats $(\mathrm{t}=6.81, \mathrm{P}<0.05 ; \mathrm{t}=4.34$, $\mathrm{P}<0.05 ; \mathrm{t}=5.46, \mathrm{P}<0.05$; Figure $4 D$ ), while inhibition of MIP$1 \alpha$ or CCR 1 following the use of miRNA-138 inhibitor significantly lowered the expressions of TNF- $\alpha$, IL- $\beta$, and IL-6 ( $\mathrm{t}=4.31, \mathrm{P}<0.05 ; \mathrm{t}=5.84, \mathrm{P}<0.05 ; \mathrm{t}=4.29, \mathrm{P}<0.05$; Figure 4C). Thus, miRNA-138 alleviated the progression of NP by lowering MIP-1 $\alpha$ and CCR1 expressions in pSNL rats.

\section{Discussion}

miRNAs play a crucial role in neuronal and immune responses (16). Among miRNAs, miRNA-138 has been reported to be involved in NP and neuroinflammation (17). In the present experiment, we observed downregulated miRNA-138 expression and upregulated TLR4 expression in the spinal cord of pSNL rats, which were accompanied by the occurrence of NP (decreased MWT and PWTL) and neuroinflammation (increased expressions of TNF- $\alpha$, IL- $\beta$, and IL- 6 ) and the activation of the MIP- $1 \alpha / C C R 1$ signaling pathway. To determine the role of miRNA-138 in NP and inflammatory response, in our current study we used LVmiRNA-138 mimic to overexpress miRNA-138. The results showed that miRNA-138 attenuated the proinflammatory response in the spinal cord, thereby ameliorating pSNLinduced NP and neuroinflammation, which was achieved by upregulating TLR4 and inhibiting the MIP-1 $\alpha / C C R 1$ signaling pathway.

Many studies have demonstrated that miRNA-138 plays key roles in the pathogenesis of prostate cancer (18), non-small cell lung cancer (19), colon cancer (20), Alzheimer's disease (21), and many other conditions. For instance, Yu et al. found that upregulation of miRNA-138 inhibited prostate cancer progression and metastasis by suppressing Wnt/ $\beta$-catenin pathway. Huang et al. showed 
that LncRNA-H19 increased cyclin-dependent kinase 1 (CDK1) expression by downregulating miRNA-138 activity, thereby promoting the proliferation of non-small cell lung cancer cells. Yang et al. demonstrated that long noncoding RNA-H19 facilitated the motility of colon cancer cells through sponging miR-138 and upregulating HMGA1 (human high mobility group A1). In our current study, we found that miRNA-138 expression was significantly downregulated after NP and neuroinflammation were induced in pSNL rats. Meanwhile, miRNA-138 overexpression significantly suppressed thermal and mechanical nociceptive responses and downregulated the protein expressions of neuroinflammation-related factors TLR4, TNF- $\alpha$, IL- $\beta$, and IL-6, suggesting miRNA-138 plays an inhibitory role in the development of NP in vivo.

TLR4 is a key member in the TLR family and plays a role in many diseases. In a recent study, TLR4 inhibition provided neuroprotection and promoted a microglial switch from the inflammatory $\mathrm{M} 1$ phenotype to the protective M2 phenotype in Alzheimer's disease. The mechanism involved may be related to modulation of the MyD88/ NF-kappaB/NLRP3 signaling pathway (22). Furthermore, traumatic brain injury (TBI) was observed to induce significant upregulation of TLR4 in the liver, with peak expression occurring 24 hours after TBI; in contrast, the hepatoprotective effects of estradiol after TBI may be mediated via the downregulation expression of TLR4. TLR4-related MAPK signaling pathway is involved in autophagy and oxidative stress in monocytes from transgenic sheep (23). In addition, overexpression of TLR4 suppresses oxidative stress and inflammatory responses and enhances autophagy (24). The roles of miRNA and TLR4 in disease development and progression are being increasingly clarified. For instance, miRNA-345-5P can alleviate the inflammatory response in mice with allergic rhinitis by inhibiting the TLR4/ NF $\kappa B$ pathway (25). Additionally, miR-155 deficiency can ameliorate pelvic tactile allodynia/ hyperalgesia in experimental autoimmune prostatitis mice and improve inflammation and oxidative stress in prostatic tissues in a TLR4-dependent manner, thereby exerting a therapeutic effect in chronic prostatitis treatment (26). In the present study, we observed the development of NP (decreased MWT and PWTL) and neuroinflammation in pSNL rats, and these changes were accompanied by the upregulation of the TLR 4 gene, suggesting that TLR4 is associated with NP and neuroinflammation. The expressions of both TLR4 and miRNA-138 were altered in the spinal cord of pSNL rats. miRNA-138 overexpression after LV use significantly downregulated the expression of TLR4 in the spinal cord of pSNL rats, suggesting the protection of miRNA-138 in NP.

MIP-1 $1 \alpha$, released by multiple immune and glial cells, is involved in the regulation of inflammatory pain and nociceptive hypersensitivity. It regulates and the inflammation and nervous system by activating CCR1, the receptor for MIP-1 $\alpha$. Our current study demonstrated that intrathecal injection of miRNA-138 significantly reduced inflammatory response and inhibited MIP-1 $\alpha /$ CCR 1 signaling pathway in pSNL rats. In addition, we found that inhibition of either MIP-1 $\alpha$ or CCR1 upregulated the inflammatory response and improved NP in rats in the pSNL + miRNA-138 inhibitor group by blocking MIP$1 \alpha$ with MIP- $1 \alpha$ neutralizing antibody (MIP- $1 \alpha \mathrm{Ab}$ ) or by using CCR1 inhibitor CCR1-HY; thus, the MIP-1 $\alpha /$ CCR1 signaling pathway is involved in the mechanism by which miRNA138 improves NP in pSNL rats in vivo.

Although there are many researches about the microRNA in the NP, we firstly reported the effect of miRNA138 in the neuroinflammation. Our experiments revealed that miRNA-138 can influence NP and neuroinflammation by regulating TLR4 in pSNL rats. Further, we verified the protection of miRNA-138 in NP and neuroinflammation was achieved by regulating MIP- $1 \alpha / C C R 1$ signaling pathway by rescue experiments. In conclusion, miRNA138 can downregulate TLR4 expression in the spinal cord of pSNL rats, inhibit the MIP-1 $\alpha / C C R 1$ signaling pathway, and ultimately alleviate NP. However, considering the role of immune cell and neurocyte in the NP, whether the miRNA138 participated in the immune cell-mediated or neurocyte-mediated inflammation still needs further exploration. Also, whether this research can be applied in human is uncertain. Our present study still lack the corresponding cell experiments and clinical statistics, which might be the future orientation.

\section{Acknowledgments}

Funding: None.

\section{Footnote}

Reporting Checklist: The authors have completed the ARRIVE reporting checklist. Available at http://dx.doi. org/10.21037/apm-21-669

Data Sharing Statement: Available at http://dx.doi. 
org/10.21037/apm-21-669

Conflicts of Interest: All authors have completed the ICMJE uniform disclosure form (available at http://dx.doi. org/10.21037/apm-21-669). The authors have no conflicts of interest to declare.

Ethical Statement: The authors are accountable for all aspects of the work in ensuring that questions related to the accuracy or integrity of any part of the work are appropriately investigated and resolved. All experimental protocols were approved by the ethics committee of Laboratory Animal Center, Hebei Medical University (approval No. 20200912) and were performed in accordance with the Guidelines for the Care and Use of Laboratory Animals in our center.

Open Access Statement: This is an Open Access article distributed in accordance with the Creative Commons Attribution-NonCommercial-NoDerivs 4.0 International License (CC BY-NC-ND 4.0), which permits the noncommercial replication and distribution of the article with the strict proviso that no changes or edits are made and the original work is properly cited (including links to both the formal publication through the relevant DOI and the license). See: https://creativecommons.org/licenses/by-nc-nd/4.0/.

\section{References}

1. Li H, Huang $\mathrm{Y}, \mathrm{Ma} \mathrm{C}$, et al. MiR-203 involves in neuropathic pain development and represses Rap1a expression in nerve growth factor differentiated neuronal PC12 cells. Clin J Pain 2015;31:36-43.

2. Blyth FM. Global burden of neuropathic pain. Pain 2018;159:614-7.

3. Sommer C, Leinders M, Üçeyler N. Inflammation in the pathophysiology of neuropathic pain. Pain 2018;159:595-602.

4. Su S, Shao J, Zhao Q, et al. MiR-30b Attenuates Neuropathic Pain by Regulating Voltage-Gated Sodium Channel Nav1.3 in Rats. Front Mol Neurosci 2017;10:126.

5. Zhu A, Shen L, Xu L, et al. Suppression of Wnt5a, but not Wnts, relieves chronic post-thoracotomy pain via antiinflammatory modulation in rats. Biochem Biophys Res Commun 2017;493:474-80.

6. Zhang Z, Gong Q, Li M, et al. MicroRNA-124 inhibits the proliferation of C6 glioma cells by targeting Smad4. Int J Mol Med 2017;40:1226-34.
7. Wang CY, Yang SH, Tzeng SF. MicroRNA-145 as one negative regulator of astrogliosis. Glia 2015;63:194-205.

8. Gong H, Song L, Lin C, et al. Downregulation of miR138 sustains NF-kB activation and promotes lipid raft formation in esophageal squamous cell carcinoma. Clin Cancer Res 2013;19:1083-93.

9. Miao W, Bao TH, Han JH, et al. Voluntary exercise prior to traumatic brain injury alters miRNA expression in the injured mouse cerebral cortex. Braz J Med Biol Res 2015;48:433-9.

10. Zhang J, Liu D, Feng Z, et al. MicroRNA-138 modulates metastasis and EMT in breast cancer cells by targeting vimentin. Biomed Pharmacother 2016;77:135-41.

11. Zhang H, Zhang H, Zhao M, et al. MiR-138 inhibits tumor growth through repression of EZH2 in non-small cell lung cancer. Cell Physiol Biochem 2013;31:56-65.

12. Zhang Y, Chen K, Sloan SA, et al. An RNA-sequencing transcriptome and splicing database of glia, neurons, and vascular cells of the cerebral cortex. J Neurosci 2014;34:11929-47.

13. Bachtell R, Hutchinson MR, Wang X, et al. Targeting the Toll of Drug Abuse: The Translational Potential of Toll-Like Receptor 4. CNS Neurol Disord Drug Targets 2015;14:692-9.

14. Rodriguez Lavado J, Sestito SE, Cighetti R, et al. Trehalose- and glucose-derived glycoamphiphiles: smallmolecule and nanoparticle Toll-like receptor 4 (TLR4) modulators. J Med Chem 2014;57:9105-23.

15. Kiguchi N, Maeda T, Kobayashi Y, et al. Macrophage inflammatory protein-1alpha mediates the development of neuropathic pain following peripheral nerve injury through interleukin-1beta upregulation. Pain 2010;149:305-15.

16. Kress $M$, Hüttenhofer A, Landry $M$, et al. microRNAs in nociceptive circuits as predictors of future clinical applications. Front Mol Neurosci 2013;6:33.

17. Zhu B, Gao J, Ouyang Y, et al. Overexpression Of miR138 Ameliorates Spared Sciatic Nerve Injury-Induced Neuropathic Pain Through The Anti-Inflammatory Response In Mice. J Pain Res 2019;12:3135-45.

18. Yu Z, Wang Z, Li F, et al. miR-138 modulates prostate cancer cell invasion and migration via $\mathrm{Wnt} / \beta$-catenin pathway. Mol Med Rep 2018;17:3140-5.

19. Huang T, Wen Y, Peng B, et al. Upregulated lncRNA H19 promotes non-small cell lung cancer cell proliferation through miR-138/PDK1 axis. Int J Clin Exp Pathol 2017;10:9012-20.

20. Yang Q, Wang X, Tang C, et al. H19 promotes the migration and invasion of colon cancer by sponging miR- 
138 to upregulate the expression of HMGA1. Int J Oncol 2017;50:1801-9.

21. Boscher E, Husson T, Quenez O, et al. Copy Number Variants in miR-138 as a Potential Risk Factor for Early-Onset Alzheimer's Disease. J Alzheimers Dis 2019;68:1243-55.

22. Cui W, Sun C, Ma Y, et al. Inhibition of TLR4 Induces M2 Microglial Polarization and Provides Neuroprotection via the NLRP3 Inflammasome in Alzheimer's Disease. Front Neurosci 2020;14:444.

23. Amiresmaili S, Khaksari M, Shahrokhi N, et al. Evolution of TLR4 role in mediating the hepatoprotective effects of estradiol after traumatic brain injury in male rats. Biochem Pharmacol 2020;178:114044.

Cite this article as: Jin $\mathrm{Y}, \mathrm{Xu} \mathrm{L}, \mathrm{Xu}$ Y. Effect of intrathecal injection of miRNA-138 on neuropathic pain in rats undergoing partial sciatic nerve ligation and its underlying mechanism. Ann Palliat Med 2021;10(6):6873-6882. doi: 10.21037/apm-21-669
24. Wang S, Song X, Zhang K, et al. Overexpression of TollLike Receptor 4 Affects Autophagy, Oxidative Stress, and Inflammatory Responses in Monocytes of Transgenic Sheep. Front Cell Dev Biol 2020;8:248.

25. Liu J, Jiang Y, Han M, et al. MicroRNA-345-5p acts as an anti-inflammatory regulator in experimental allergic rhinitis via the TLR4/NF- $\mathrm{KB}$ pathway. Int Immunopharmacol 2020;86:106522.

26. Fu X, He HD, Li CJ, et al. MicroRNA-155 deficiency attenuates inflammation and oxidative stress in experimental autoimmune prostatitis in a TLR4dependent manner. Kaohsiung J Med Sci 2020;36:712-20.

(English Language Editor: J. Gray) 